\title{
Front Matter: Volume 9907
}

, "Front Matter: Volume 9907," Proc. SPIE 9907, Optical and Infrared Interferometry and Imaging V, 990701 (11 November 2016); doi: $10.1117 / 12.2254897$

Event: SPIE Astronomical Telescopes + Instrumentation, 2016, Edinburgh, SPIE. United Kingdom 


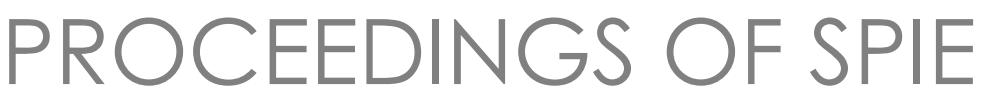

\title{
Optical and Infrared Interferometry and Imaging $V$
}

\author{
Fabien Malbet \\ Michelle J. Creech-Eakman \\ Peter G. Tuthill \\ Editors
}

27 June - 1 July 2016

Edinburgh, United Kingdom

Sponsored by

SPIE

Cooperating Organizations

American Astronomical Society (United States) - Australian Astronomical Observatory (Australia) - Association of Universities for Research in Astronomy (AURA) - Canadian Astronomical Society (CASCA) (Canada) - Canadian Space Agency (Canada) • European Astronomical Society (Switzerland) - European Southern Observatory (Germany) • National Radio Astronomy Observatory - Royal Astronomical Society (United Kingdom) • Science \& Technology Facilities Council (United Kingdom)

Published by

SPIE

Volume 9907

Proceedings of SPIE 0277-786X, V. 9907

SPIE is an international society advancing an interdisciplinary approach to the science and application of light.

Optical and Infrared Interferometry and Imaging V, edited by Fabien Malbet,

Michelle J. Creech-Eakman, Peter G. Tuthill, Proc. of SPIE Vol. 9907, 990701

(C) 2016 SPIE · CCC code: 0277-786X/16/\$18 - doi: 10.1117/12.2254897 
The papers in this volume were part of the technical conference cited on the cover and title page. Papers were selected and subject to review by the editors and conference program committee. Some conference presentations may not be available for publication. Additional papers and presentation recordings may be available online in the SPIE Digital Library at SPIEDigitallibrary.org.

The papers reflect the work and thoughts of the authors and are published herein as submitted. The publisher is not responsible for the validity of the information or for any outcomes resulting from reliance thereon.

Please use the following format to cite material from these proceedings:

Author(s), "Title of Paper," in Optical and Infrared Interferometry and Imaging $V$, edited by Fabien Malbet, Michelle J. Creech-Eakman, Peter G. Tuthill, Proceedings of SPIE Vol. 9907 (SPIE, Bellingham, WA, 2016) Six-digit Article CID Number.

ISSN: 0277-786X

ISSN: 9781510601949 (electronic)

ISBN: 9781510601932

Published by

SPIE

P.O. Box 10, Bellingham, Washington 98227-0010 USA

Telephone +1 3606763290 (Pacific Time) · Fax +1 3606471445

SPIE.org

Copyright (C) 2016, Society of Photo-Optical Instrumentation Engineers.

Copying of material in this book for internal or personal use, or for the internal or personal use of specific clients, beyond the fair use provisions granted by the U.S. Copyright Law is authorized by SPIE subject to payment of copying fees. The Transactional Reporting Service base fee for this volume is $\$ 18.00$ per article (or portion thereof), which should be paid directly to the Copyright Clearance Center (CCC), 222 Rosewood Drive, Danvers, MA 01923. Payment may also be made electronically through CCC Online at copyright.com. Other copying for republication, resale, advertising or promotion, or any form of systematic or multiple reproduction of any material in this book is prohibited except with permission in writing from the publisher. The CCC fee code is 0277-786X/16/\$18.00.

Printed in the United States of America.

Publication of record for individual papers is online in the SPIE Digital Library.

\section{SPIE. DIGITAL}

Paper Numbering: Proceedings of SPIE follow an e-First publication model. A unique citation identifier (CID) number is assigned to each article at the time of publication. Utilization of CIDs allows articles to be fully citable as soon as they are published online, and connects the same identifier to all online and print versions of the publication. SPIE uses a six-digit CID article numbering system structured as follows:

- The first four digits correspond to the SPIE volume number.

- The last two digits indicate publication order within the volume using a Base 36 numbering system employing both numerals and letters. These two-number sets start with 00, 01, 02, 03, 04, $05,06,07,08,09,0 A, 0 B \ldots$. OZ, followed by 10-1Z, 20-2Z, etc. The CID Number appears on each page of the manuscript. 


\title{
Contents
}

\author{
xi Authors \\ xvii Conference Committee \\ xxi Introduction
}

\section{Part One}

\section{FACILITIES I}

990702 The Navy Precision Optical Interferometer: an update (Invited Paper) [9907-1]

990703 An update on the CHARA array (Invited Paper) [9907-2]

990704 Overview of LBTI: a multipurpose facility for high spatial resolution observations (Invited Paper) [9907-3]

\section{FACILITIES II}

990705 A new path to first light for the Magdalena Ridge Observatory interferometer (Invited Paper) [9907-4]

990706 The 2nd generation VLTI path to performance (Invited Paper) [9907-5] INSTRUMENTS

9907 OA An overview of the mid-infrared spectro-interferometer MATISSE: science, concept, and current status (Invited Paper) [9907-9]

9907 OB Imaging capabilities of the VLTI/MATISSE spectro-interferometric instrument (Invited Paper) [9907-10]

9907 OC MATISSE: alignment, integration, and test phase first results [9907-11]

\section{SCIENCE I}

9907 OD Imaging protoplanets: observing transition disks with non-redundant masking (Invited Paper) [9907-12] 
APERTURE MASKING I

9907 OE A conceptual scheme for cophasing across gaps in segmented pupils with a laser guide star Fizeau interferometer (Invited Paper) [9907-13]

SPECKLE INTERFEROMETRY

9907 0J Speckle imaging at large telescopes: current results and future prospects (Invited Paper) [9907-18]

9907 OK SRAO: the first southern robotic AO system [9907-19]

\section{SCIENCE II}

9907 OL The role of Fizeau interferometry in planetary science [9907-20]

INTENSITY INTERFEROMETRY

9907 OM Intensity interferometry: optical imaging with kilometer baselines (Invited Paper) [9907-21]

9907 ON Intensity interferometry with Aqueye+ and Iqueye in Asiago [9907-22]

9907 OP On the measurement of intensity correlations from laboratory and astronomical sources with SPADs and SNSPDs [9907-24]

SCIENCE III

$99070 Q \quad$ The orbit of the mercury-manganese binary 41 Eridani [9907-25]

SCIENCE IV

9907 OR Infrared interferometry and AGNs: Parsec-scale disks and dusty outflows (Invited Paper) [9907-26]

\section{SPACE INTERFEROMETRY}

9907 OS The path to interferometry in space (Invited Paper) [9907-27]

9907 OT Optics of Balloon Experimental Twin Telescope for Infrared Interferometry (BETTII): delay lines and alignment [9907-28]

9907 OU Recent experiments conducted with the Wide-field imaging interferometry testbed (WIIT) [9907-29]

iv 
9907 OV The innermost astronomical units of protoplanetary disks (Invited Paper) [9907-30]

\section{DATA PROCESSING I}

9907 0X Making high-accuracy null depth measurements for the LBTI exozodi survey [9907-32]

$99070 Z$ Direct temperature map estimation in optical long baseline interferometry [9907-138]

\section{DATA PROCESSING II}

990710 OIFITS version 2: the new standard for optical/IR interferometry data exchange (Invited Paper) [9907-34]

990711 ASPRO2: get ready for VLTI's instruments GRAVITY and MATISSE [9907-35]

990712 Spectrally dispersed Fourier-phase analysis for redundant apertures [9907-36]

\section{SCIENCE VI}

990713 Enabling the direct detection of earth-sized exoplanets with the LBTI HOSTS project: a progress report [9907-37]

SCIENCE VII

990714 Imaging transient events at high angular resolution (Invited Paper) [9907-38]

\section{DETECTORS FOR INTERFEROMETRY}

990715 Fast sub-electron detectors review for interferometry (Invited Paper) [9907-39]

990716 Progress towards photon-counting infrared arrays for interferometry [9907-40]

\section{SCIENCE VIII}

990717 Interferometric studies of disk-eclipsed binary star systems (Invited Paper) [9907-41] 


\section{ASTROPHOTONICS}

990718 Astronomical photonics in the context of infrared interferometry and high-resolution spectroscopy (Invited Paper) [9907-42]

990719 Increasing the spectral coverage of interferometric integrated optics: K/L and N-laserwritten beam combiners [9907-43]

9907 1A ALOHA project: how nonlinear optics can boost interferometry to propose a new generation of instrument for high-resolution imaging [9907-44]

\section{SCIENCE IX}

9907 1B Evolved stars at high angular resolution: present and future (Invited Paper) [9907-45]

INTERFEROMETRY IMAGING

9907 1C Interferometric image reconstruction: techniques, results, and future direction (Invited Paper) [9907-46]

9907 1D The 2016 interferometric imaging beauty contest (Invited Paper) [9907-47]

9907 1E Global optimization for image restoration in optical/IR interferometry [9907-48]

\section{FRINGE TRACKING}

9907 if Hierarchical fringe tracker to co-phase and coherence very large optical interferometers [9907-49]

9907 IG Simultaneous water vapor and dry air optical path length measurements and compensation with the large binocular telescope interferometer [9907-50]

\section{FUTURE INSTRUMENTS}

$99071 \mathrm{H}$ Long baseline interferometry in the visible: first results of the FRIEND project [9907-51]

\section{PLANET FORMATION IMAGER}

9907 IK Planet Formation Imager (PFI): science vision and key requirements [9907-54]

9907 IL Status of the Planet Formation Imager (PFI) concept (Invited Paper) [9907-55] 


\section{Part Two}

PLANET FORMATION IMAGER II

9907 1N Beam combination schemes and technologies for the Planet Formation Imager [9907-57]

990710 Architecture design study and technology road map for the Planet Formation Imager (PFI) [9907-58]

WEDNESDAY POSTER SESSION

9907 IQ How to create space inside the VLTI: PIONIER 3D project [9907-60]

9907 is Fundamental gain in high-contrast imaging with the large binocular telescope interferometer [9907-62]

9907 IT Sensitivity to differential piston and to adaptive optics errors with the Large Binocular Telescope Interferometer [9907-63]

9907 IW SCSI: the Southern Connecticut Stellar Interferometer [9907-66]

9907 1X Monitoring a decade of seeing at the NPOI with quad cell measurements [9907-67]

$99071 Z$ VLT interferometer upgrade for the 2nd generation of interferometric instruments [9907-69]

990720 NAOMI: a low-order adaptive optics system for the VLT interferometer [9907-70]

990721 Control bandwidth improvements in GRAVITY fringe tracker by switching to a synchronous real time computer architecture [9907-71]

990722 The metrology system of the VLTI instrument GRAVITY [9907-73]

990723 Data reduction for the MATISSE instrument [9907-74]

990724 ALOHA/CHARA at $1.55 \mu \mathrm{m}$ : sensitivity improvement and on-sky ability to detect astronomical sources in $\mathrm{H}$ band [9907-75]

990725 The new classic instrument for the navy precision optical interferometer [9907-76]

990727 GRAVITY acquisition camera: characterization results [9907-78]

990728 MATISSE: specifications and expected performances [9907-79]

$99072 \mathrm{~B} \quad \mathrm{H}_{\infty}$ controller design for high sensitivity fringe tracking [9907-82]

9907 2C Multi-baseline chain bootstrapping with new classic at the NPOI [9907-83]

9907 2E C-RED One: the infrared camera using the Saphira e-APD detector [9907-86]

9907 2F GRAVITY detector systems [9907-87] 
$99072 \mathrm{G}$ Measuring the thermal sensitivity of a fiber Fabry-Pérot interferometer [9907-88]

$99072 \mathrm{H} \quad$ Experimental demonstration of a crossed cubes nuller for coronagraphy and interferometry [9907-89]

9907 2l Khayyam: progress and prospects of coupling a spatial heterodyne spectrometer (SHS) to a Cassegrain telescope for optical interferometry [9907-90]

9907 2K Results from a multi aperture Fizeau interferometer ground testbed: demonstrator for a future space-based interferometer [9907-93]

9907 2L Fiber-based heterodyne infrared interferometry: an instrumentation study platform on the way to the proposed Infrared Planet Formation Imager [9907-94]

990720 Recent developments with the visible nulling coronagraph [9907-98]

\section{THURSDAY POSTER SESSION}

$99072 R \quad$ Differential speckle and wide-field imaging for the Gemini-North and WIYN telescopes [9907-100]

9907 2T Sparse aperture masking with SPHERE [9907-102]

$99072 \mathrm{U}$ Interferometric direct imaging properties of a BIGRE-DAM device in laboratory [9907-103]

990730 Chalcogenide glass planar MIR couplers for future chip based Bracewell interferometers [9907-109]

990731 All-in-one 4-telescope beam combination with a zig-zag array of waveguides [9907-110]

990732 ALOHA @3,39 $\mu \mathrm{m}$ : implementation of the up-conversion interferometer in the L band [9907-111]

990733 6- and 8-telescope discrete beam combiners [9907-112]

990734 Model-based calculations of fiber output fields for fiber-based spectroscopy [9907-1 13]

990735 Use of a photonic lantern into an image plane fiber beam combiner [9907-1 14]

990736 Fringe tracking at longer wavelengths using near- and mid-IR integrated optics devices [9907-115]

$990738 \quad$ Novel multi-telescopes beam combiners for next generation instruments (FIRST/SUBARU) [9907-1 17]

990739 Recent results on photonic devices made by laser writing: 3D 3T near IR waveguides, midIR spectrometers and electro-optic beam combiners [9907-118]

9907 3B Interferometric field of view measurements at the VLTI [9907-120]

9907 3D Effective a posteriori co-phasing of interferometric fringe data [9907-122] 
9907 3E P-REx: the piston drift reconstruction experiment [9907-123]

9907 3F Image restoration for a hypertelescope [9907-124]

9907 3G Rotation and translation registration of bandlimited interferometric images using a chirp ztransform [9907-125]

$99073 \mathrm{H} \quad$ Image reconstruction method IRBis for optical/infrared long-baseline interferometry [9907-126]

9907 3l High fidelity imaging of geosynchronous satellites with the MROI [9907-127]

9907 3K Interbands phase models for polychromatic image reconstruction in optical interferometry [9907-129]

9907 3N User-friendly imaging algorithms for interferometry [9907-132]

9907 3P An interferometric view of binary stars [9907-135]

$99073 Q \quad$ Grown-up stars physics with MATISSE [9907-136]

9907 3R Sharp images of WR104 [9907-137]

9907 3S Science with MATISSE [9907-139]

9907 3T Observing the PTPS sample of evolved exoplanet host candidates using the NPOI [9907-140]

9907 3V First year report of the Optical Interferometry DataBase [9907-142]

9907 3W Co-phasing the planet formation imager [9907-144]

9907 3X Practical beam transport for PFI [9907-145]

990740 The Wide-field Imaging Interferometry Testbed (WIIT): recent progress in the simulation and synthesis of WIIT data [9907-148]

990741 Stray light evaluation for the astrometric gravitation probe mission [9907-149]

990742 AGP (Astrometric Gravitation Probe) optical design report [9907-150] 
Proc. of SPIE Vol. 9907 990701-10

Downloaded From: https://www.spiedigitallibrary.org/conference-proceedings-of-spie on 25 Apr 2023 Terms of Use: https://www.spiedigitallibrary.org/terms-of-use 


\section{Authors}

Numbers in the index correspond to the last two digits of the six-digit citation identifier (CID) article numbering system used in Proceedings of SPIE. The first four digits reflect the volume number. Base 36 numbering is employed for the last two digits and indicates the order of articles within the volume. Numbers start with 00, 01, 02, 03, 04, 05, 06, 07, 08, 09, OA, OB...0Z, followed by 10-12, 20-2Z, etc.

\begin{tabular}{|c|c|}
\hline Abad, José Antonio, $1 Z$ & Berger, Jean-Philippe, 06,0A, $1 \varepsilon$ \\
\hline Abadie, Sergio, $1 \mathrm{Z}$ & 20 \\
\hline Absil, Olivier, OS, OX & Berggren, Karl, OP \\
\hline Abuter, Roberto, 12, 21 & Bério, Philippe, OA, OC, 15, 1H, 2 \\
\hline Accardo, M., OC & Besser, Felipe E., 2L \\
\hline Acuña, Margarita, $1 \mathrm{Z}$ & Bettonvil, F., OA, OC \\
\hline Ade, Peter, OT & Beuzit, Jean-Luc, 20, 2T \\
\hline Agabi, Karim, $1 \mathrm{~F}$ & Bitsch, Bertram, iK \\
\hline Agocs, T., OA, OC & Blind, Nicolas, 22 \\
\hline Aller-Carpentier, Emmanuel, 20 & Bodenmüller, Daniel, 34 \\
\hline Allouche, Fatmé, $0 A, 0 C, 1 Z, 23,28$ & Boffin, Henri M. J., 3P \\
\hline Alonso Herrero, Almudena, $1 \mathrm{~K}$ & Böhm, M., $1 G$ \\
\hline Alonso, Jaime, 06, 1Z, 20 & Bolados, Carlos, $1 \mathrm{Z}$ \\
\hline Amorim, Antonio, 22, 27 & Bolcar, Matthew R., OU, 20, 40 \\
\hline Andolfalto, Luigi, 1z, 20 & Bonnet, Henri, 06, 1 Z \\
\hline Anselmi, Alberto, 42 & Bonsor, Amy, 1K \\
\hline Antichi, Jacopo, $2 U$ & Boskri, Abdelkarim, 1F, 2B, 3W \\
\hline Antonelli, Pierre, 0A, 0C, 1Z, 28 & Bourdarot, Guillaume, $2 \mathrm{H}$ \\
\hline Anugu, Narsireddy, 27 & Bourgès, Laurent, 11, 28, 3V \\
\hline Arcidiacono, Carmelo, 1S, $1 \mathrm{~T}$ & Bourget, Pierre, $0 \mathrm{~A}, 1 \mathrm{Q}, 1 \mathrm{Z}$ \\
\hline Arezki, Brahim, $2 \mathrm{H}$ & Boutolleau, David, 2E \\
\hline Armitage, Phil, $1 \mathrm{~K}$ & Boyajian, Tabetha, $1 \mathrm{~K}$ \\
\hline Armstrong, J. Thomas, 02, 1X, 25, 2C, 3T & Brandner, Wolfgang, 22, 27 \\
\hline Arriola, Alexander, 19 & Brast, Roland, OA, OC, $1 \mathrm{Z}$ \\
\hline Augereau, Jean-Charles, OA, 3S & Bresson, Yves, OA, OC, 1F, $1 \mathrm{H}$ \\
\hline Avila, Gerardo, OA, $1 Z$ & Briguglio, Runa, OD \\
\hline Baba, Naoshi, 3F & Bristow, Paul, OA, OC, 12 \\
\hline Baccani, Cristian, 41 & Bryan, Sean, OP \\
\hline Baccichet, Nicola, 2K & Bryden, Geoffrey, 0X, 13 \\
\hline Bailet, C., OA, OC, $1 \mathrm{H}$ & Burtscher, Leonard, OR \\
\hline Bailey, Vanessa P., 04, 0D, 0X, 13 & Buscher, David F., 05, 16, 1K, 31 \\
\hline Baines, Ellyn K., 02, 25, 2C, 3T & Busonero, Deborah, 42 \\
\hline Baker, Ian, 2E & Caillat, Amandine, 2K \\
\hline Barbier, D., 38 & Caniguante, Luis, IZ \\
\hline Barbieri, Cesare, $0 \mathrm{~N}$ & Cassagnettes, C., 38 \\
\hline Barbieri, Mauro, ON & Castillo, Roberto, $1 \mathrm{Z}$ \\
\hline Baron, Fabien, OB, 1C, 10 & Cervantes, R., 05 \\
\hline Barriga, Pablo José, 12 & Cesare, Stefano, 42 \\
\hline Baruteau, Clement, IK & Cheetham, Anthony C., 2T \\
\hline Bate, Matthew R., $1 \mathrm{~K}$ & Chiavassa, Andrea, $1 \mathrm{~K}, 3 \mathrm{Q}, 3 \mathrm{~S}$ \\
\hline Bayo, Amelia, 1K & Choudhury, Debaditya, 19 \\
\hline Bazin, G., 28 & Ciardi, David, IK \\
\hline Beckmann, U., OA, 0C, 23 & Clampin, Mark, 20 \\
\hline Behrend, J., OA & Clark, James H., 02 \\
\hline Beltran, Juan, 1 Z & Clausse, J.-M., OA, OC, $1 \mathrm{H}$ \\
\hline Bendjoya, P., 3Q & Clop, Fabien, 2E \\
\hline Benisty, Myriam, 0V, 1K, 3V & Close, Laird M., OD \\
\hline Benkhaldoum, Zouhair, IF & Connot, C., OA \\
\hline Benson, James A., 02, 3T & Conrad, Albert R., OL \\
\hline
\end{tabular}


Conzelmann, Ralf, OA, $1 \mathrm{Z}$

Correia, Jean-Jacques, 20

Cortes, Angela, 1Z, 20

Creech-Eakman, Michelle J., 05, 10, 31

Cruzalèbes, P., OA, OC, 23, 28, 3Q

Csepany, G., OA

Cvetojevic, Nick, 18, 30

Dahl, C., 05

Dalgarno, Paul A., 35

d'Amico, C., 39

Danchi, William C., 04, 0A, 0X, 13, $1 G$

Darré, Pascaline, 1A, 24, 32

De Haan, M., OA

de Wit, Willem-Jan, 06, 1K, 12

Debbarma, S., 30

Defrère, Denis, 04, OD, 0S, 0X, 13, $1 \mathrm{G}$

Dejonghe, J., $1 \mathrm{H}$

Del Valle, Diego, $1 \mathrm{Z}$

Delage, Laurent, 1A, 24, 32

Delbo, Marco, OA, 3S

Delboulbé, Alain, 20

Delplancke-Strobele, Françoise, 1Z, 20

Dembet, Roderick, 21

Derie, Frederic, $1 \mathrm{Z}$

Dhabal, Arnab, OT

di Lieto, Nicola, 21

Diaz, Alvaro, $1 \mathrm{Z}$

Diddams, Scott A., $2 \mathrm{G}$

Diener, Romina, 19, 31, 36

DiMaio, Alex J., IW

Dohlen, Kjetil, 2K

Domiciano de Souza, A., 3Q

Dominik, Carsten, 0A, 3S

Donaldson, Rob, 20

Dong, Ruobing, 10

Donoso, Reinaldo, 12

Dorn, Reinhold J., 1Z, 20

Dow, Thomas, OT

Downey, E., 04, $1 \mathrm{G}$

Dravins, Dainis, OM

Duchêne, Gaspard, $1 \mathrm{~K}$

Duhoux, Philippe, $1 \mathrm{Z}$

Dupuy, Christophe, 1Z, 20

Durney, O., 04, 1G

Duvert, Gilles, 0Q, 10, 11, 3D, 3N, 3V

Eggleton, B., 30

Egner, Sebastian E., 06, 1Z, 20

Eisenhaver, Frank, 06, 1Z, 21, 22, 27, 2F

Eisner, Josh, OD

El Halkouj, Thami, 1F, 2B, 3W

Elao, Christian, 12

Elswijk, E., OA, OC

Errmann, Ronny, 33

Ertel, S., 04, 13, 1G

Espaillat, Catherine, $1 \mathrm{~K}$

Esposito, Simone, 04, 1S, IT

Fantei, Y., OA

Fantei-Caujolle, Y., OC

Farinato, Jacopo, $2 \mathrm{U}$

Farisato, Giancarlo, ON
Farrington, Chris D., 03, 3V

Farris, A., 05

Favazza, Paolo, ON

Feautrier, Philippe, 15, 2E

Fedou, Pierre, 21

Fernandez, Ruben, $1 z$

Ferrari, André, OZ, 1D, 3K

Fienup, James R., OU, $3 G$

Finger, Gert, OA, 16, 2F

Fisher, M., 05

Fixsen, Dale, OT

Focardi, Mauro, 41

Folcher, Jean-Pierre, 1F, 2B

Follette, Katherine B., OD

Frahm, R., OC

Fu, Qiang, 3E

Fuenteseca, Eloy, 12

Furst, Stephen, OT

Gabasch, A., OA

Gach, Jean-Luc, 15, 2E

Gai, Mario, 41, 42

Gallenne, Alexandre, $1 \mathrm{~K}$

Gallieni, Daniele, 42

Gandhi, Poshak, 1K

García López, Rebeca, OV

Garcia, Paulo, 27, 3N

Gardillou, F., 38

Gaytan, Daniel, 1 Z

Genzel, Reinhard, 22

Gies, D. G., 03

Gillessen, Stefan, 22

Girard, Julien, 2T, 2U

Giro, Enrico, 2U

Giudice, Andrea, 1W

Glindemann, Andreas, 0A, 0C, 1 Z

Gomes, Jean-Thomas, 1 A

Gonté, Frédéric Yves J., 06, 0A, 1Q, 1Z, 20

Gonzáles Herrera, J.-C., OC

Gonzales, Jaime, 1 z

Gonzalez, Jean-François, $1 \mathrm{~K}$

Gordo, Paulo, 27

Graser, U., OA, OC

Gratton, Raffaele, $2 \mathrm{U}$

Greenaway, Alan H., 35

Greffe, Timothée, 2E

Grenz, P., 04

Grossard, Ludovic, 1A, 24, 32

Guieu, Sylvain, 06, 1Q, 1 Z

Guisard, Stephane, $1 \mathrm{Z}$

Guitton, F., OA, 23

Gulinatti, Angelo, 1W

Guniat, S., OA

Guyot, C., 38

Haguenaver, Pierre, 06, 0A, 1Q, 12

Haimerl, Andreas, 12

Halverson, Samuel, $2 \mathrm{G}$

Hanenburg, H., OA

Haniff, Christopher A., 05, 13, 1K, 1N, 31

Hans, Oliver, 22

Harries, Tim J., $1 \mathrm{~K}$ 
Harris, Robert, 18

Harris, Walter, 21

Haubois, Xavier, 2T, 3V

Hauden, J., 38

Haug, Marcus, 22

Haußmann, Frank, 22

Haynes, Roger, 18

Heininger, Matthias, OA, 0C, 1D, 23, 3H

Heinz, Volker, 1 Z

Helmbrecht, Michael A., 20

Hénault, François, 2H

Henning, Thomas, OA, OB, 3S

Henriquez, Juan Pablo, 12

Hernandez, Eloy, 34

Herrmann, Harald, 32

Hicks, Brian A., 20

Hill, John M., 04, 1G, 1S, $1 T$

Hindsley, R., 25

Hinz, Philip M., 04, 0D, 0X, 13, 1G, 1S, 1T

Hofferbert, R., OA

Hoffmann, W. F., 04, IG

Hofmann, Karl-Heinz, OA, OB, 1D, 23, 3H, 3Q, 3R 3S

Hogerheijde, Michiel, OA, 3S

Holland, W., OS

Hönig, Sebastian, OR, $1 \mathrm{~K}$

Horch, Elliott P., OJ, 1W, 2R

Hosseini, Sona, 2

Howard, Joseph M., 20

Howell, Steve B., 2R

Hron, Josef, $0 A, 3 Q, 3 S$

Huber, David, 22

Huber, Stefan, 20

Hubin, Norbert, 1Z, 20

Hubrig, Swetlana, $O Q$

Huby, E., 38

Huerta, Rodrigo, 12

Hummel, Christian A., OA, OQ, 10, 3B

Hutter, Donald J., 02

lacchetta, Alexander S., OU, 3G, 40

llee, John, $1 \mathrm{~K}$

Ireland, Michael J., 03, 1K, 1L, 1N, 10, 30, 3W, 3X

Isderda, J., OA

Isella, Andrea, 1L, 10

Ives, D., OA, OC

Jaffe, Walter, OA, OC, OR, 23, 3S

Jakob, G., OA

Jasko, A., OA

Jenka, L., 05

Jennings, Jeff, $2 G$

Jensen, Eric L. N., $1 \mathrm{~K}$

Jochum, Lieselotte, $1 \mathrm{Z}$

Jocou, L., IQ

Jofre, Mauricio, $2 \mathrm{~L}$

Johnston, P., 05

Jolley, Paul, OA, 20

Jorgensen, A. M., 25, $2 \mathrm{C}$

Jovanovic, Nemanja, 18

Juanola-Parramon, Roser, OS, OU, 40

Juhasz, Attila, 1K, 3S
Kane, Stephen R., $1 \mathrm{~K}$

Kellner, Stefan, 22

Kelly, R., 05

Kelz, Andreas, 34

Kenchington Goldsmith, H.-D., 30

Kennedy, Grant M., OX, 13

Khorrami, Z., 3Q

Kiraly, S., OA

Kirchbauer, Jean-Paul, 20

Kishimoto, Makoto, OR, $1 \mathrm{~K}$

Kley, Wilhelm, $1 \mathrm{~K}$

Klinglesmith, D., 05

Kluska, Jacques, OV, $0 Z$

Kok, Yitping, 22

Kragt, J., OA

Kral, Quentin, 1K

Kratschmann, Tobias, 22

Kratter, Kaitlin, OD, 1K

Kraus, Stefan, 1K, 1L, 10, 3W

Kroener, T., OA

Kroes, G., OA

Kuindersma, S., OA

Labadie, Lucas, OA, 18, 19, 1K, 1N, 31, 36

Lacour, Sylvestre, 18, 1K, 1N, 21, 2T, 38

Lagadec, E., 3R

Lagarde, Stephane, OA, OC, IF, 23, 28

Landini, Federico, 41, 42

Lanz, Thierry, OA, 3Q, 3S

Lasen, Matias, $2 \mathrm{~L}$

Lattanzi, Mario Gilberto, 42

Laughlin, Greg, $1 \mathrm{~K}$

Laun, W., OA, OC

Law, Nicholas M., OK

Lawrence, J. S., 30

Lazzarini, Paolo, 42

Le Bouquin, Jean-Baptiste, 0 Q, 1Q, 20, 3B, 3V

Le Louarn, Miska, 20

Léger, Jonathan, $1 \mathrm{E}$

Lehmitz, M., OA, OC

Leinert, C., OA

Leisawitz, David T., OS, OU, 3G, 40

Leisenring, J., 04

Leiva, Alfredo, 12

Lemarchand, Stephane, 2E

Lessio, Luigi, ON

Lévêque, Samuel, 12

Lilley, Paul, 20

Lippa, Magdalena, 22

Lizon, Jean-Louis, OA, 0C, $1 \mathrm{Z}$

Lopez, Bruno, OA, OB, OC, 23, 28, 3Q, 3R, 3S

Lopez-Gonzaga, Noel, OR

Love, H., 05

Luco, Fernando, $1 Z$

Lucuix, C., OA

Luther-Davies, B., 30

Lyon, Richard G., 20

Ma, P., 30

Macintosh, Bruce A., OD

MacPherson, William N., 19

Madden, Gillian, 19 
Madden, S. J., 30

Magnard, Yves, 20

Mahadevan, Suvrath, $2 \mathrm{G}$

Maher, Stephen F., OU, 40

Males, Jared R., OD

Mani, Hamdi, OP

Marcos, Michel, 2K

Marcotto, A., OA, OC

Mardones, Pedro, $1 \mathrm{Z}$

Marion, Lindsay, $\mathrm{OX}$

Martin, Guillermo, 18, 38, 39

Martinache, Frantz, OA, 12

Martinod, M. A., 1H

Martinot-Lagarde, G., OA

Martis, Alessandro, 20

Mary, David, 1D, 3K

Masset, Frederic, $1 \mathrm{~K}$

Matter, Alexis, OA, OB, OC, 1K, 23, 28, 3Q, 3R, 3S

Mauclert, N., OA

Maurel, Didier, 20

Mauskopf, Philip, OP

McAlister, H. A., 03

Mehrgan, Leander $\mathrm{H} ., \mathrm{OA}, 2 \mathrm{~F}$

Meilland, Anthony, OA, OC, 1H, 23, 3Q, 3S

Meisenheimer, Klaus, OA, OR, 3S

Meixner, K., OC

Mella, Guillaume, 3N, 3V

Mellado, Angel, 12

Mellein, M., OA, OC

Menardi, S., OA

Mennesson, Bertrand, 04, 0X, 13, 1G

Mentzell, Eric, OT

Mérand, Antoine, 06, 0A, 10, 1Q, 12, 3B

Meru, Farzana, $1 \mathrm{~K}$

Michael, Ernest A., 1L, 1O, 2L

Michaud, Laurence, 20

Millan-Gabet, Rafael, 04, 0X, 13, 1G, 1K

Miller, Ian J., 20

Millour, Florentin, OA, OB, OC, 1D, 1H, 1K, 23, 28,

$3 \mathrm{H}, 3 \mathrm{Q}, 3 \mathrm{R}, 3 \mathrm{~S}$

Minardi, Stefano, 18, 19, 1L, 1N, 10, 31, 33, 36

Miura, Noriaki, 3F

Monnier, John D., 03, 1K, 1L, 10, 3W

Montoya, M., 04, 1G

Morbidelli, Alessandro, $1 \mathrm{~K}$

Mordasini, Chris, $1 \mathrm{~K}$

Morel, S., OA, OC

Morlok, Andreas, $1 \mathrm{~K}$

Morozov, Dmitry, OP

Morzinski, Katie M., 04, OD

Moulin, Thibaut, 20

Mourard, Denis, 1H, 2U, 3V

Mozurkewich, David, OS, 1L, 10, 25, 2C, 3X

Mundy, Lee, OT

Murakami, Naoshi, 3F

Muthusubramanian, Balaji, 19, 36

Nakai, Yuto, 3F

Naletto, Giampiero, ON

Nardetto, Nicolas, 3Q, 35

Nelson, Richard P., 1K
Neumann, U., OA, OC

Niccolini, G., 3Q

Niedzielski, Andrzej, 3T

Nolte, Stefan, 19, 31

Nowak, Matthias, OX

Nusdeo, Daniel A., IW

Nussbaum, E., OA

Ochoa, D., 05

Olivares, A., 05

Olofsson, Johan, $1 \mathrm{~K}$

Ortega, Nicolas, 2L

Osorio, Juan, $1 \mathrm{Z}$

Ott, Jürgen, $1 \mathrm{Z}$

Ott, Thomas, 22, 27

Ottogalli, S., OA

Oudmaijer, Rene, $1 \mathrm{~K}$

Ozon, Matthew, IE

Paardekooper, Sijme-Jan, $1 \mathrm{~K}$

Packham, Chris, $1 \mathrm{~K}$

Paladini, Claudia, 1B, 1K, 3Q, 3S

Pallanca, Laurent, $1 Z$

Palsa, R., OA

Pancrazzi, Maurizio, 41

Panduro, Johana, OA, 2F

Panic, Olja, IK

Pantin, Eric, OA, 35

Pasquini, Luca, 1Z, 20

Patru, Fabien, 1S, 1T, 2U

Paufique, Jérôme, 20

Pavez, Marcus, 12

Payne, Ifan, 05, 31

Pedretti, Ettore, 35

Pepper, Joshua, $1 \mathrm{~K}$

Percheron, Isabelle, 0A, $1 \mathrm{Z}$

Peronio, Pietro, $1 \mathrm{~W}$

Perraut, Karine, 1H, 22, 27

Perrin, Guy, 18, 22, 27

Peter, Diethard, 3E

Petrone, Peter, 20

Petrov, Romain G., OA, OC, 1F, 1K, 1L, 1O, 23, 28,

2B, 3Q, 3S, 3W

Pettazzi, Lorenzo, 06

Pfuhl, Oliver, 22, 27

Phan Duc, Thanh, OA, 1Z, 20, 21

Pierens, Arnaud, $1 \mathrm{~K}$

Pijoan, Jordi, $1 \mathrm{~J}$

Pilyavsky, Genady, OP

Pina, Miguel I., 2 L

Pineda, Juan Carlos, 12

Pinna, Enrico, 04, 1S, $1 T$

Pino, Andres, 12

Pino, J., 05

Pinte, Christophe, $1 \mathrm{~K}$

Pirard, Jean-Francois, 12

Plattner, Markus, 22

Pollarolo, Clemente, 2L

Pope, Benjamin, IK

Pott, Jörg-Uwe, OA, OB, 19, 1G, 1K, 36, 3E

Poupar, Sébastien, 06, 1Q, 12

Pozna, E., OA 
Price, J., 05

Puglisi, Alfio T., 04, 0D, 1S, $1 \mathrm{~T}$

Pugnat, T., 38

Quentin, Jutta, 20

Quiroga-Nuñez, Luis Henry, 1K

Rabou, Patrick, $2 U$

Rakotonimbahy, Eddy, 2K

Ramírez, Andres, $1 \mathrm{Z}$

Ramos Almeida, Cristina, $1 \mathrm{~K}$

Ramos, Nicolas, $2 \mathrm{~L}$

Rates, Alfredo, 2L

Rau, Christian, 22

Raymond, Sean, $1 \mathrm{~K}$

Rea, A., 05

Rech, Ivan, IW

Regaly, Zsolt, IK

Reinero, Claudio, 12

Restaino, Sergio R., 02, $1 X$

Reyes, Javier, 20

Reynaud, François, 1A, 24, 32

Reynolds, Mark, 1K

Riccardi, Armando, 1S, IT

Ridgway, Steve T., 03, 1K

Ridings, Robert, 20

Rieke, G., 13

Riker, J., 05

Rinehart, Stephen A., OS, OT, OU, 1L, 10, 40

Riquelme, Miguel, $1 \mathrm{Z}$

Riva, Alberto, 41, 42

Rivinius, Thomas, 1Q, 1 Z

Rizzo, Maxime J., OS, OT

Robbe-Dubois, S., OA, 0C, 23, 28

Roberge, Aki, OX, 13

Rochat, Sylvain, 20

Rochelle, S., 05

Rodenas, A., 39

Rodigas, Timothy J., OD

Roelfsema, R., OA

Rojas, Chester, $1 \mathrm{Z}$

Romero, Juan, 12

Romero, V., 05

Rosotti, Giovanni, 1K

Roth, Martin M., 18, 34

Roux, Alain, 20

Rozas, Felix, 12

Rupert, Justin D., 1W

Rupprecht, G., OA

Salcido, C., 05

Salgado, Fernando, $1 \mathrm{Z}$

Sallum, Steph, OD

Sampler, Henry, OT

Sanchez-Bermudez, J., OB, ID

Santoro, F., 05

Savini, Giorgio, 0S, 2K, 40

Schaefer, Gail H., 03, 14

Scheithaver, Silvia, 12

Schertl, Dieter, OA, OB, 1D, 23, 3H, 3Q, 3S

Schmid, Christian, $1 \mathrm{Z}$

Schmidt, C., OA

Schmidt, L., 05
Schmitt, Henrique R., 02, 1X, 25, 2C, 3T

Schoeller, M., OA

Schöller, Markus, 06, OC, 0Q, 12

Schroeder, Edward, OP

Schuhler, Nicolas, 06, 1Q, 12

Schuil, M., OA

Schutz, Antony, 0Z, 1D, 3D, 3K

Schworer, Guillaume, 2T

Scott, Nicholas J., 03, 24, 2R

Seneta, Eugene B., 05, 16

Serabyn, Eugene, 0X, 13

Shchkaturov, Pavel, 20

Siclari, Waldo, 12

Silberhorn, Christine, 32

Silverberg, Robert F., OT

Sinclair, Adrian, $\mathrm{OP}$

Skemer, Andrew J., 04, 0D, 0X, 13, 1G

Skrutskie, M., 04

Smirnov, Konstantin, OP

Smith, Michael, $1 \mathrm{~K}$

Smith, Nathan, $O P$

Soulain, A., 23, 3Q, 3R

Soulez, Férréol, OZ, 1E, 3D, 3K

Spalding, Eckhart, 04, 0D, 1G

Spang, Alain, $\mathrm{OA}, 1 \mathrm{H}, 2 \mathrm{H}$

Spencer, L., OS

Stadler, Eric, 20, 2E

Stapelfeldt, Karl, 0X, 13

Stassun, Keivan, $1 \mathrm{~K}$

Stee, P., 3Q

Stegmeier, J., OA

Stencel, Robert E., 17

Stephan, Christian, 12

Stoian, R., 39

Stone, J., 04, $1 \mathrm{G}$

Straubmeier, Christian, 22, 27

Sturm, Eckhard, 22

Sturmann, J., 03, 24

Sturmann, L., 03

Suarez, Marcos, 20

Sun, Xiaowei, 05, 16

Surdej, Jean, $1 \mathrm{~K}$

Szemendera, Ludovic, 1A, 32

Tallon, Michel, OZ, 1H, 3D

Tallon-Bosc, Isabelle, 1H, 3D

Tamblay, Richard, 12

Tamura, Motohide, 3F

Tapia, Mario, $1 \mathrm{Z}$

ten Brummelaar, Theo A., 03, 1K, 1L, 10, 24, 3V

Tepper, Jan, 19, 31, 36

ter Horst, R., OA

Terrien, Ryan, $2 \mathrm{G}$

Thévenin, F., 3Q

Thiébaut, Éric, OZ, 1D, 1E, 3D, 3K, 3N

Thomson, Robert R., 18, 19, 1N, 35

Tintori, Matteo, 42

Tokovinin, Andrei, OK

Tristram, Konrad R. W., OR, 1K, 12

Tromp, N., OA

Tucker, Carole, OT 
Turner, N. H., 03

Turner, Neal, $1 \mathrm{~K}$

Tuthill, Peter G., OD, OE, 1K, 30

Umbriaco, Gabriele, $0 \mathrm{~N}$

Vakhtomin, Yuriy, OP

Vakili, F., OA, 3Q

Valdes, Guillermo, 1Z, 20

van Belle, Gerard T., 02, 0A, 1K, 1L, 1X, 25, 2C

van Boekel, Roy, OA, OB, 3S

van der Heyden, P., 12

van Duin, A., OA

Vanko, Martin, 3T

Vannier, Martin, OA, 1D, 3K, 3N

Vasisht, Gautam, 1K, 1L, 10

Vassallo, Daniele, $2 \mathrm{U}$

Vaz, Amali, 04, OD, 13, $1 \mathrm{G}$

Vázquez de Aldana, J. R., 39

Veach, Todd, OT

Vecchiato, Alberto, 42

Venema, L., OA

Vérinaud, Christophe, 20, 2U

Verroi, Enrico, ON

Wagner, K., OA

Waisberg, Idel, 22

Weber, Johannes, 22

Weigelt, G., OA

Weigelt, Gerd, OB, 1D, 1K, 23, 3H, 3Q, 3S

Weinberger, Alycia J., OD, 0X, 13

Weiss, Samuel A., IW

Widmann, Felix, 3E

Wieprecht, Ekkehard, 22, 27

Wiezorrek, Erich, 22, 27

Wishnow, Ed, 10

Wittkowski, Markus, $1 \mathrm{~K}$

Woillez, Julien, 06, 0A, 1L, 1Z, 20, 21

Wolf, Sebastian, 0A, 1K, 35

Wolszczan, Aleksander, $3 T$

Wright, Andrew, 12

Wyatt, Mark, OX, 13

Xomperio, Marco, OD

Yañez, Claudio, 2L

Yazıcı, Şenol, 22

Ycas, Gabriel, 2G

Young, John S., 05, 10, 16, 1D, 1L, 10, 3I, 3N, 3X

Zampieri, Luca, ON

Zavala, Robert T., 02, 3T

Zhao, Ming, $1 \mathrm{~K}$

Zhu, Di, OP

Zhu, Zhaohuan, 1K, 10

Ziegler, Carl, OK

Zielinski, Pawel, 3T

Zins, Gerard, 1Q, 1 Z 


\title{
Conference Committee
}

\author{
Symposium Chairs \\ Colin Cunningham, UK Astronomy Technology Centre \\ (United Kingdom) \\ Masanori lye, National Astronomical Observatory of Japan (Japan) \\ Symposium Co-chairs \\ Allison A. Barto, Ball Aerospace \& Technologies Corporation \\ (United States) \\ Suzanne K. Ramsay, European Southern Observatory (Germany) \\ Conference Chairs \\ Fabien Malbet, Institut de Planétologie et d'Astrophysique de \\ Grenoble (France) \\ Michelle J. Creech-Eakman, New Mexico Institute of Mining and \\ Technology (United States) \\ Peter G. Tuthill, The University of Sydney (Australia) \\ Conference Program Committee
}

Ellyn K. Baines, U.S. Naval Research Laboratory (United States)

Fabien Baron, Georgia State University (United States)

Jean-Philippe Berger, European Southern Observatory (Germany)

Elliott P. Horch, Southern Connecticut State University (United States)

Takayuki Kotani, National Astronomical Observatory of Japan

(Japan)

Lucas Labadie, Universität zu Köln (Germany)

Keiichi Ohnaka, Universidad Católica del Norte (Chile)

Claudia Paladini, Université Libre de Bruxelles (Belgium)

Jayadev K. Rajagopal, National Optical Astronomy Observatory

(United States)

Andrew Skemer, The University of Arizona (United States)

Isabelle Tallon-Bosc, Centre de Recherche Astronomique de Lyon (France)

\section{Session Chairs}

1 Facilities I

Ellyn K. Baines, U.S. Naval Research Laboratory (United States)

2 Facilities II

Ellyn K. Baines, U.S. Naval Research Laboratory (United States) 
3 Instruments

David F. Buscher, University of Cambridge (United Kingdom)

4 Science I

Fabien Baron, Georgia State University (United States)

5 Aperture Masking I

Fabien Baron, Georgia State University (United States)

6 Aperture Masking II

Fabien Baron, Georgia State University (United States)

$7 \quad$ Speckle Interferometry

Andrew J. Skemer, University of California, Santa Cruz (United States)

8 Science II

Andrew J. Skemer, University of California, Santa Cruz (United States)

9 Intensity Interferometry

Elliott P. Horch, Southern Connecticut State University (United States)

10 Science III

Elliott P. Horch, Southern Connecticut State University (United States)

11 Science IV

Lucas Labadie, Universität zu Köln (Germany)

12 Space Interferometry

Lucas Labadie, Universität zu Köln (Germany)

13 Science $V$

Henrique R. Schmitt, U.S. Naval Research Laboratory (United States)

14 Data Processing I

Henrique R. Schmitt, U.S. Naval Research Laboratory (United States)

15 Data Processing II

Henrique R. Schmitt, U.S. Naval Research Laboratory (United States)

16 Science VI

Henrique R. Schmitt, U.S. Naval Research Laboratory (United States)

Poster Previews

Fabien Malbet, Institut de Planétologie et d'Astrophysique de Grenoble (France)

xviii 
17 Science VII

Michelle J. Creech-Eakman, New Mexico Institute of Mining and Technology (United States)

18 Detectors for Interferometry

Michelle J. Creech-Eakman, New Mexico Institute of Mining and Technology (United States)

19 Science VIII

John D. Monnier, University of Michigan (United States)

20 Astrophotonics

John D. Monnier, University of Michigan (United States)

21 Science IX

Gilles Duvert, Institut de Planétologie et d'Astrophysique de Grenoble (France)

22 Interferometry Imaging

Gilles Duvert, Institut de Planétologie et d'Astrophysique de Grenoble (France)

23 Fringe Tracking

Christopher A. Haniff, University of Cambridge (United Kingdom)

24 Future Instruments

Christopher A. Haniff, University of Cambridge (United Kingdom)

25 Planet Formation Imager

Peter G. Tuthill, The University of Sydney (Australia)

26 Planet Formation Imager II

Peter G. Tuthill, The University of Sydney (Australia)

27 Interferometry Community

Michelle J. Creech-Eakman, New Mexico Institute of Mining and Technology (United States)

Closing Remarks

Michelle J. Creech-Eakman, New Mexico Institute of Mining and Technology (United States) 
Proc. of SPIE Vol. $9907990701-20$

Downloaded From: https://www.spiedigitallibrary.org/conference-proceedings-of-spie on 25 Apr 2023 Terms of Use: https://www.spiedigitallibrary.org/terms-of-use 


\section{Introduction}

Optical and infrared interferometry continues to rapidly develop and deliver unique scientific reach to astrophysics. As astronomy, in general, goes through a transformative phase, our field has encountered both opportunities and challenges. The primary goals of this conference were to allow the attendees to learn firsthand about the exciting capabilities operational at facilities across the globe and to discuss the future prospects for interferometry in technology and instrumentation. Here, the SPIE meeting plays a unique role as the singular venue that brings together interferometrists from the world over.

For this meeting in Edinburgh, key themes were the burgeoning scientific productivity encapsulated in many invited reviews, the emergence of new instrumentation (particularly from photonics), and the continuing development of image reconstruction techniques. Closure-phase measurements and nulling results from large aperture telescopes continue to help push astrophysical investigations of fainter objects. New instrumentation will bring all these aspects together in the very near future, with increasing interest from non-specialists in interferometry thanks to general user facilities and data-reduction software.

This conference has primarily focused on the latest innovations in technology and engineering for ground- and space-based interferometry in the optical and infrared, including new instrumentation, techniques and software. For example, we had many contributions on integrated optics and detectors. Marquee scientific results in areas ranging from young stellar objects to active galactic nuclei have been made possible with milli- and micro-arcsecond angular resolution.

In several important domains, the distinction between interferometry and aperture masking has been dissolving for some years. The newest generation of extremely large telescopes occupy some middle ground where the field of high-resolution imaging with a single aperture-traditionally aperture masking or speckle interferometry-has been powerfully augmented by the advent of high-performing adaptive optics. Although the major surge in interest in space interferometry from the previous decade has abated, there are several smaller missions from balloons to cubesats that are generating significant interest and keeping these ideas alive.

We had an interesting community discussion that allowed introspection and brainstorming for the intermediate and long-term future of interferometry. Scientific and technical opportunities, priorities and new directions in times of limited funding were major themes. This volume presents a total of 102 contributions (a similar total to recent meetings - 96 in 2012, 116 in 2014).

We would like to thank all oral contributors, invited talks and posters who made this conference an important milestone in our domain.

\section{Fabien Malbet \\ Michelle J. Creech-Eakman Peter G. Tuthill}


Proc. of SPIE Vol. 9907 990701-22

Downloaded From: https://www.spiedigitallibrary.org/conference-proceedings-of-spie on 25 Apr 2023 Terms of Use: https://www.spiedigitallibrary.org/terms-of-use 